\title{
In vivo expression of proteases and protease inhibitor, a serpin, by periodontal pathogens at teeth and implants
}

Martin Eckert ${ }^{1}$, Danuta Mizgalska ${ }^{2}$, Anton Sculean $^{1}$, Jan Potempa ${ }^{2,3}$, Andreas Stavropoulos ${ }^{4}$, Sigrun Eick ${ }^{1}$

${ }^{1}$ Department of Periodontology, University of Bern, School of Dental Medicine, Bern, Switzerland

${ }^{2}$ Department of Microbiology, Faculty of Biochemistry, Biophysics, and Biotechnology, Jagiellonian University, Krakow, Poland

${ }^{3}$ Department of Oral Immunology and Infectious Diseases, University of Louisville School of Dentistry, Louisville, KY, USA

${ }^{4}$ Department of Periodontology, Faculty of Odontology, Malmö University, Malmö, Sweden

Running title: Bacterial proteases in vivo

Keywords: periodontal disease; peri-implant disease; Porphyromonas gingivalis; Tannerella forsythia; bacterial proteases; miropin

Correspondence: Sigrun Eick, Department of Periodontology, School of Dental Medicine, University of Bern, Freiburgstrasse 7, CH-3010 Bern, Switzerland, Tel. +4131632 2542, Fax: +41 31632 4915, e-mail: sigrun.eick@zmk.unibe.ch 


\section{SUMMARY}

Porphyromonas gingivalis and Tannerella forsythia secrete proteases, gingipains and KLIKKproteases. In addition, T. forsythia produces a serpin (miropin) with broad inhibitory spectrum. The aim of this pilot study was to determine the level of expression of miropin and individual proteases in vivo in periodontal and peri-implant health and disease conditions. Biofilm and gingival/peri-implant crevicular fluid (GCF or PISF respectively) samples from healthy tooth and implant sites $(n=10)$, gingivitis and mucositis sites $(n=12)$, and periodontitis and peri-implantitis sites ( $n=10$ ). Concentration of interleukins (IL)-8, IL-1 $\beta$ and IL-10 in GCF was determined by ELISA. Loads of $P$. gingivalis and $T$. forsythia and the presence of proteases and miropin genes were assessed in biofilm by qPCR, while genes expression was estimated by qRT-PCR.

Presence of $P$. gingivalis and $T$. forsythia, as well as the level of IL-8 and IL-1 1 , were associated with disease severity in the periodontal and peri-implant tissues. In biofilm samples harboring $T$. forsythia genes encoding proteases were found to be present at $72.4 \%$ for karilysin and $100 \%$ for other KLIKK-proteases genes and miropin. At the same time, detectable mRNA expression of individual genes was in the range from $20.7 \%$ to $58.6 \%$ samples (for forsylisin and miropsin-1, respectively). In comparison to the $T$. forsythia proteases, miropin and the gingipains were highly expressed. The level of expression of gingipains was associated with those of miropin and certain $T$. forsythia proteases around teeth but not implants. Cumulatively, KLIKK-proteases and especially miropin might play a role in pathogenesis of both periodontal and peri-implant diseases. 


\section{INTRODUCTION}

According to an emerging paradigm, pathogenesis of periodontal disease is considered as a result of disturbed homeostasis within tooth-supporting tissues by dysbiotic microbiota leading to inflammation and slowly eroding periodontal tissues. ${ }^{1}$ Using murine model of diseases it was shown that the presence of certain bacteria might influence the entire microbiome around the tooth by modulating the immune response, thus leading to a shift from a symbiotic into a dysbiotic microbiota. ${ }^{1}$ In particular, $P$. gingivalis was designated as a keystone pathogen in this transition. ${ }^{2}$ The virulence of $P$. gingivalis heavily relies on its arginine- and lysine-specific cysteine proteases, called gingipains. ${ }^{3,4}$ The arginine-specific gingipains (RgpA and RgpB) are encoded by two genes, $\operatorname{rgp} A$ and $\operatorname{rgp} B$. The lysine-specific gingipain (Kgp) originates from a single gene, $\mathrm{kgp}^{5}$ Gingipains can skew the immune response by cleaving immunoglobulin $\mathrm{G} 1$, interfering with the complement system, and disassembling cytokine cross-talk networks. ${ }^{6,7}$

Apart from $P$. gingivalis other bacterial species such as $T$. forsythia may play a similar key role. $T$. forsythia, together with $P$. gingivalis, is a part of the so called red complex, ${ }^{8}$ and they are very often found together in sites with periodontal destruction. ${ }^{9,} 10$ For example, $T$. forsythia was detected in approximately $50 \%$ of young adult patients with gingivitis, ${ }^{11}$ while in $85 \%$ of cases in subjects with chronic periodontitis often (50\% cases) in association with $P$. gingivalis. ${ }^{10}$ However, little is still known regarding the virulence factors of $T$. forsythia. The genome of $T$. forsythia contains many sequences that apparently encode proteases. ${ }^{12}$ In particular, 6 enzymes with a KLIKK sequential motive at the C-terminus and therefore referred to as KLIKK-proteases are of interest as putative virulence factors. ${ }^{12}$ Interestingly, $T$. forsythia also secretes miropin, a potent protease inhibitor belonging to the serpin superfamily, which may contribute to pathogenicity. The unique feature of miropin is the ability to inhibit a broad range of target proteases, including neutrophil-derived cathepsin $G$ and elastase important for antibacterial activity of these phagocytes. ${ }^{13,14}$

During the last 2-3 decades, replacement of teeth with implants became a predictable and widely used therapy. However, peri-implant diseases are now becoming increasingly prevalent and negatively impacting oral health. In a recent systematic review, the weighted mean prevalence of peri-implant mucositis and peri-implantitis among individuals with implants was $43 \%$ and $22 \%$, respectively. ${ }^{15}$ Despite major similarities in the pathogenesis of peri-implant diseases with that of periodontal diseases, there are apparent differences in the host response to the bacterial challenge in these diseases. ${ }^{16}$ For example, $T$. forsythia appears to play a greater role in pathogenesis of peri-implant disease than in periodontitis. Ten years after placement of implants, greater numbers of $T$. forsythia were found at implant 
sites than at the adjacent teeth in correlation with disease severity. ${ }^{17}$ The tissue destruction in peri-implantitis seems to be more progressive and extensive than in periodontitis. ${ }^{18}$ So far, however, it is not known which $T$. forsythia putative virulence factors are expressed in vivo and whether there are differences in their expression between periodontal and peri-implant diseases and/or disease severity.

Thus, the aim of this pilot study was to investigate expression of $T$. forsythia miropin and KLIKK-proteases in vivo in periodontal and peri-implant diseases, correlate differences in their expression with the disease entity, and determine a possible association between expression of $T$. forsythia and $P$. gingivalis proteases in sites infected with both pathogens.

\section{MATERIALS AND METHODS}

\section{Subject recruitment}

Thirty-two subjects were recruited from patients attending the specialty clinic at the Department of Periodontology, Faculty of Odontology, Malmö University, Malmö, Sweden. These subjects contributed with periodontally healthy sites and healthy implants $(n=10)$, with gingivitis and mucositis sites $(n=12)$, as well as with periodontitis and peri-implantitis sites $(n=10)$.

Periodontal diseases (gingivitis, periodontitis) were defined based on the classification system established in $1999,{ }^{19}$ while peri-implant diseases (mucositis and peri-implantitis) were defined according to Zitzmann and Berglundh. ${ }^{20}$ An ethical approval was granted by the Regional Ethics Committee in Lund, Sweden (Dr. nr.; 2014/700), and all patients signed an informed written consent prior to entering the study.

\section{Sampling}

Samples were obtained from the site of the tooth or implant with the deepest pocket depth, according to existing dental record registrations, using paper points and paper strips. The periodontal or peri-implant probing depths were $<4 \mathrm{~mm}$ in periodontal health, $3-5 \mathrm{~mm}$ in gingivitis, 3-6 mm in peri-implant-health or mucositis, $5-9 \mathrm{~mm}$ in case of periodontitis and 6 - $9 \mathrm{~mm}$ in peri-implantitis lesions. For collecting gingival crevicular fluid (GCF) or peri-implant sulcular fluid (PISF) the site was gently air dried and isolated by cotton rolls; then paper strips were inserted in the entrance of the sulcus (superficial method) ${ }^{21}$ for $30 \mathrm{~s}$. This procedure was immediately followed by collection of subgingival biofilm. For this, endodontic paper points were inserted into pockets until resistance from the base of the pocket was felt and were kept in place for $30 \mathrm{~s}$. After sampling, paper strips and points were stored in sterile 
tubes. The tubes for storing paper points also contained $300 \mu \mathrm{l}$ of RNAlater (RNAlater®, Sigma-Aldrich, St. Louis, USA). Paper points and strips were stored shortly after collection at $-80^{\circ} \mathrm{C}$ until processed.

\section{Levels of GCF/PISF biomarkers}

GCF/PISF samples were eluted in $750 \mu$ phosphate-buffered saline (PBS) overnight at $4^{\circ} \mathrm{C}$. The levels of interleukin (IL)-8, IL-1 $\beta$ and IL-10 in the GCF/PISF were determined by using commercially available ELISA kits (DuoSet® ELISA Development Systems kits (R\&D Systems, Inc., Minneapolis, Minnesota, USA) according to the manufacturer's instructions. The detection levels were $1 \mathrm{pg} / \mathrm{site}$ each.

\section{DNA/RNA extraction}

DNA and RNA were extracted simultaneously from paper points using a DNA/RNA extraction kit (innuPREP DNA/RNA Mini Kit, Analytik Jena, Jena, Germany) according to the manufacturer's instructions.

\section{Determination of bacterial counts}

To determine counts of bacteria being associated with periodontitis ( $P$. gingivalis, $T$. forsythia, Treponema denticola, Aggregatibacter actinomycetemcomitans), the real-time polymerase chain reaction (PCR) was performed using the 7500 Real-time PCR System (Applied Biosystems ${ }^{\mathrm{TM}}$, Foster City, USA) and reference strains as described previously. ${ }^{22}$ The detection level was determined as $10^{3}$ bacteria per sample.

\section{Expression of bacterial proteases and miropin}

To determine the in vivo expression of $T$. forsythia proteases miropsin-1, miropsin-2, mirolysin, mirolase, karilysin and forsylisin-1, the protease inhibitor miropin, and the gingipains $r g p A, \operatorname{rgpB}$ and $\mathrm{kgp}, \mathrm{cDNA}$ was amplified from total RNA obtained from the paper points. The RNA was first treated with DNase I, RNase-free (ThermoFisher Scientific, Waltham, Massachusetts, USA) and thereafter cDNA was generated using RevertAid Reverse Transcriptase (ThermoFisher Scientific) according to the manufacturer's instructions.

Real-time PCR using GoTaq ${ }^{\circledR}$ qPCR Master Mix (Promega) and the respective primers (Table 1) was performed for different bacterial proteases and miropin according to the 
manufacturer's instructions. Quantification was made related to the sod gene expression of respective bacteria.

Negative controls contained 1:5 diluted RNA (before generation of cDNA) and as positive controls cDNA generated from RNA of $24 \mathrm{~h}$-cultures of $P$. gingivalis ATCC 33277 as well as of $T$. forsythia ATCC 43037 were used.

\section{Statistics}

Statistical analysis was performed using non-parametric tests (Kruskal-Wallis, MannWhitney, Friedman tests) for continuous variables and $\mathrm{Chi}^{2}$-test for dichotomized variables, with SPSS 23.0 (IBM, Chicago, IL, USA).

\section{RESULTS}

\section{Presence of bacteria and biomarkers levels in gingival / peri-implant fluid}

There were no statistically significant differences between biofilms collected from the teeth and implants in terms of frequency of detection and numbers of the various bacteria at health and at the various disease severities. In both cases bacterial presence increased from health to disease in correlation with severity of periodontal and peri-implant destruction (Table 2). The differences between healthy and diseased sites were statistically significant for $T$. forsythia and T. denticola, both, at the teeth $(p=0.024 ; p=0.007)$ and at implants $(p=0.011$; $\mathrm{p}=0.004)$.

In GCF collected from periodontal sulci or pockets around teeth, the level of IL-8 correlated with the severity of periodontal disease $(p=0.048)$, and it was significantly higher in periodontitis than in gingivitis $(p=0.036)$ or in health $(p=0.029)$. Amount of IL-1 $\beta$ in GCF also varied in a manner dependent on the periodontal disease severity $(p=0.010)$. At teeth, levels of IL-1 $\beta$ were higher in periodontitis than in gingivitis $(p=0.004)$, whereas at implants there was no statistically significant difference between mucositis and peri-implantitis but only between the health and mucosistis sites $(p=0.025)$ and between the health and periimplantitis sites $(p=0.043)$. Comparing teeth with implants, the levels of IL-1 $\beta$ in periodontitis exceeded those in peri-implantitis $(p=0.035)$. The anti-inflammatory $I L-10$ was lower in periodontitis and peri-implantitis in comparison with gingivitis $(p=0.021)$ and mucositis $(p<0.001)$. Results with statistically significant differences are presented in Figure 1. 


\section{Porphyromonas gingivalis and expression of gingipains}

Expression of gingipains genes was standardized on expression of the housekeeping gene sod. In samples with $P$. gingivalis counts less than $10^{5}$ we sometime failed to detect the sod transcript therefore we have compared gingipain expression levels only in samples with $\geq 10^{5}$ bacteria per site. In this subset of samples expression of each gingipain was always detectable, with $\mathrm{kgp}$ expression being the highest, followed by $\operatorname{rgp} A$ and $\operatorname{rgp} B(p<0.001)$ (Table 3).

\section{Tannerella forsythia and expression of its proteases and miropin}

The part of samples bearing less than $10^{5} T$. forsythia were excluded from quantitative analysis because no mRNA for the sod gene could be detected (Table 4). In the subset of biofilm samples with bacterial load $>10^{5}$ the detection of the protease genes varied from $72.4 \%$ (karilysin) to $100 \%$ (miropsin-2, mirolysin, mirolase, miropin) of the samples. Detectable amount of mRNA of protease genes transcripts was found at lower frequency ranging from $20.7 \%$ (forsylisin-1) to $58.6 \%$ (miropsin-1) of the samples. Quantitatively except for one peri-implantitis specimen having high level of miropsin-2, in all other samples expression of any protease gene was always low (in comparison to sod).

In T. forsythia-positive samples (bacterial count over $10^{5}$ ) there was no statistically significant difference in detection of protease gene transcripts in samples collected from the tooth and implant sites (Table 5). Exception was miropsin-2 showing higher levels of expression in periodontitis or peri-implantitis than in periodontal health/gingivitis or peri-implant health/mucositis (teeth: $p=0.015$; implants: $p=0.044$ ).

Contrary to the proteases, miropin was highly expressed. In all samples positive for $T$. forsythia, miropin was detected on the mRNA level. In samples with $\geq 10^{5} \mathrm{~T}$. forsythia expression was in median 2.34 related to sod (Table 4).

\section{Correlation of proteases expressions}

Expression of the three gingipains was highly correlated, both at teeth ( $r=0.890$ up to $r=0.972$, each $p<0.001)$ and implants biofilms $(r=0.996$ up to $r=0.999$, each $p<0.001)$. Also expression of $T$. forsythia proteases was correlated, with the strongest correlation seen for karilysin and forsylisin- $1(r=0.856, p<0.001)$, then miropsin- 1 and mirolase $(r=0.769, p<0.001)$ in biofilm derived for the subgingival teeth surface. In biofilm collected from the implant sites the strongest correlation was found between mirolase and miropsin-2 $(r=0.796, p<0.001)$ and then between mirolase and miropsin-1 $(r=0.790, p<0.001)$. In biofilm collected from teeth also 
the miropin expression was found to correlate with those of miropsin-1 $(r=0.740, p<0001)$ and miropsin-2 $(r=0.784, p<0.001)$. In case of the biofilm from implants the correlation factor $r$ did not exceed 0.6 for any combination of $T$. forsythia protease genes expression.

In biofilm collected from tooth sites, gingipains mRNA levels correlated with expression of the $T$. forsythia proteases. The strongest correlation was found between $\operatorname{rgp} B$ or $\mathrm{kgp}$ with miropsin-2 ( $r=0.523, p=0.002 ; r=503, p=0.003$, respectively) and between kgp and mirolase $(\mathrm{r}=0.501, \mathrm{p}=0.003)$. There was also a positive correlation between gingipains and miropin expressions (rgpA: $r=0.536, p=0.002$; $r g p B: r=0.429, p=0.014$, kgp: $r=0.550, p=0.001$ ).

In biofilm from implant sites, no significant correlation between gingipains and any of $T$. forsythia proteases was detected ( $r$ was always below 0.5). The same was observed for relation between the miropin and gingipain expression.

In samples collected from teeth sites, gingipain expression in biofilm did not correlate with the levels of biomarkers in GCF. In contrast, the expression of certain T. forsythia proteases correlated positively with the IL-1 $\beta$ GCF level. This was found for miropsin-1 ( $r=0.417$; $\mathrm{p}=0.018)$ and karilysin $(\mathrm{r}=0.406, \mathrm{p}=0.021)$. In samples from implant sites, expression of miropsin-1 was positively associated with levels of $\mathrm{IL}-1 \beta(\mathrm{r}=0.417, \mathrm{p}=0.018)$ and negatively associated with those of IL-10 ( $r=-0.430, p=0.014)$. Also the expression of miropsin-2 $(r=-$ $0.391, p=0.027)$ and mirolase $(r=-0.510, p=0.003)$ were inversely correlated with $\mathrm{IL}-10$ levels in PISF. No association of the miropin expression with the biomarker levels was observed.

Found correlations were underlined by a principal components analysis, where components with more than $20 \%$ variance were considered (Table 6). Principal components analysis is a multivariate statistical method used to find hidden complex, and possible relationships between features in a data set. Correlated features are converted by means of the principal axes transformation into new features, the so-called 'principal components' where the importance of a factor (variable) is expressed by its loading. The principal components themselves are uncorrelated. ${ }^{23}$

The clear association between certain $T$. forsythia proteases is visible both in the teeth- and implants-derived biofilms. In this analysis, miropin has only a relevant loading at teeth, and biomarkers (IL-8, IL-1 $\beta$ ) are linked with karilysin expression also in the teeth-surface biofilm.

\section{DISCUSSION}

In this study, the presence and expression of $P$. gingivalis and $T$. forsythia proteases and the bacterial protease inhibitor, miropin, was assessed in biofilm samples derived from teeth and implants, in health and disease. Of note, instead the 16S RNA gene known to differ in number of copies even within one species ${ }^{24}$ we chose sod as a reference gene. Being aware 
that sod expression depends on oxygen tension ${ }^{25}$ we assumed that sampled sites were rather uniform with respect to anaerobic conditions and small variations in oxygen tension should not significantly affect local sod expression. The above threshold level of mRNA of bacterial sod was reliably and quantitatively measured in all samples having a bacterial load equal or higher than $10^{5}$ bacteria per site. Groups were differentiated related to the degree of inflammation without alveolar bone loss and showing different levels of pathological bone destruction. Both pro-inflammatory biomarkers, IL-8 and IL-1 $\beta$ increased from health to disease, and with disease severity, i.e. from gingivitis/mucositis to periodontitis/periimplantitis. On the other hand, the level of the anti-inflammatory IL-10 was high at inflamed sites and low at sites exhibiting bone destruction, both at implants and at the teeth. The finding of higher levels of IL-1 $\beta$ and lower levels of IL-10 at peri-implantitis sites is in accordance with the observation by Casado et al. ${ }^{26}$ Interestingly, in contradiction to the observation by Hultin et al. ${ }^{27}$ showing similar levels of this cytokine in sites with periimplantitis and periodontitis collected from the same patient, we have found lower levels of IL-1 $\beta$ at peri-implantitis sites than in GCF collected from periodontitis sites. This discrepancy suggests that more detailed studies need to be performed to differentiate importance of cytokines in the pathology of peri-implantitis and periodontitis.

As expected, disease severity was associated with an increased prevalence of certain bacterial species, i.e. a significantly larger numbers of $T$. forsythia and $T$. denticola were found at periodontitis/peri-implantitis sites compared to gingivitis/mucositis sites, and in gingivitis/mucositis sites they were more abundant than in healthy sites. However, there was never a difference between the bacterial counts at the teeth and implants, collected from sites of the comparable severity, i.e. gingivitis versus peri-implant mucositis, and periodontitis versus peri-implantitis. In general, our finding of correlation between the pathogens load and the disease severity is in accordance with results reported by Cortelli et al. ${ }^{28}$ In this study authors counted periodontal pathogens collected from around teeth and implants from two independent groups of individuals. They found lower amount of $T$. denticola and $T$. forsythia at implants in comparison with the teeth. ${ }^{28}$ This observation contradicts our finding but the discrepancy must be due to different experimental set-up. On the other hand, the fact that $T$. denticola and $T$. forsythia can easily transmit from a periodontally diseased tooth to an implant $^{29-31}$ and probably vice versa, explains the comparable load of these periodontal pathogens around the diseased teeth and implants herein.

P. gingivalis cysteine proteases (gingipains) were always expressed both at implants and at teeth, which corroborates with results of our recent in vitro study using titanium and dentine disks. ${ }^{32}$ The highest mRNA expression related to sod was found for $k g p$, followed by $\operatorname{rgpA}$ 
and $\mathrm{rgpB}$. Level of Kgp was determined as being up to $10 \mathrm{nM}$ in periodontium, ${ }^{33}$ those of Arg-gingipains up to $1.5 \mu \mathrm{M}$. ${ }^{34}$ Unfortunately, there is still a little data on gingipains level at peri-implantitis sites. DNA of $\operatorname{rgp} A, \operatorname{rgp} B$ and $\mathrm{kgp}$ was used as vaccines in an animal model with $\mathrm{kgp}$ being most efficiently blocking bone loss in experimental peri-implantitis whereas rgpB was ineffective.$^{35}$ Gingpains are able to cleave adherence junctions of epithelial cells, ${ }^{36}$ a property which may impair epithelial barrier around implants. Moreover, gingipaindependent manipulations of recruitment and functions of neutrophils, ${ }^{37}$ exploitation of the complement system, activation of the kinin system leading to activation of prostaglandin in osteoblasts and extravasation, ${ }^{6}$ might be of importance both at the teeth and implants.

Expression of $T$. forsythia proteases was found at lower level than that of gingipains. Protease genes transcripts were determined in $20-59 \%$ of the samples with $T$. forsythia load exceeding $\geq 10^{5}$ bacterial cells. This result confirms findings of our recent study on patients with periodontitis, in which the in vivo expression of karilysin and mirolysin was experimentally verified in a small set of samples. ${ }^{38,39}$ In the present study, mainly transcripts (mRNA) of miropsin-1, miropsin-2 and mirolase were found. In general, expression of $T$. forsythia proteases, in particular miropsin-2 was associated with periodontal or peri-implant destruction supporting a potential role of the KLIKK-proteases in disease progression. There is little known about miropsin-1 and miropsin-2 up to now. Miropsin-1 and miropsin-2 are proteolytically active when using casein or gelatine as substrates, however their activity was lower when compared to other KLIKK proteases. ${ }^{40}$ Nevertheless, they may specifically target molecules important for local homeostasis in periodontal tissues. In contrast to other KLIKK proteases, miropsin-2 is not auto-processing into lower molecular mass forms. ${ }^{40}$ Finally, mirolase was characterized as a calcium-dependent serine protease with the ability to degrade fibrinogen and hemoglobin. ${ }^{12}$

Karilysin and mirolysin expression was found in about $40 \%$ of the samples with more than $10^{5}$ T. forsythia. Karilysin was characterized as a matrix metalloprotease-like enzyme able to degrade elastin, fibrinogen and fibronectin. ${ }^{41}$ The pathogenic potential of karylisin to interfere with innate immunity is manifested by its ability to inactivate the antimicrobial peptide LL-37 ${ }^{42}$ and to induce expression of TNF $\alpha$ in macrophages, and then shedding it from the macrophage surface thus leading to an increased release of this proinflammatory cytokine. ${ }^{43}$ Similar to karilysin, the metalloprotease mirolysin can also inactivate LL-37.44 Both karilysin and mirolysin inhibit all pathways of the complement system. ${ }^{38,39}$ Expression of $T$. forsythia proteases was highly correlated at the teeth and furthermore associated also with gingipains expression. Synergism between gingipains and T. forsythia has been investigated. Gingipains are involved in a synergistic increase of IL-6 production of macrophage-like cells 
when infected with $T$. forsythia and $P$. gingivalis. ${ }^{45}$ Gingipains enhance phagocytosis of $T$. forsythia, but diminish their killing by macrophages. ${ }^{46}$ Further research should focus on the potentially synergistic role of KLIKK proteases and gingipains in pathogenesis of periodontal and peri-implantitis diseases.

Miropin, a protease inhibitor belonging to the serpin superfamily was highly expressed. The miropin mRNA level in tested samples was higher than that of sod, the house-keeping gene used as the reference. Bacterial serpins are mainly found in commensals. Eubacterium sireaum, an inhabitant of the human gut, synthesizes serpins which inhibit neutrophil elastase, protease 3 and certain gut proteases and in this way may contribute to homoeostasis in the gut. ${ }^{47}$ Also miropin is the very potent inhibitor of serine endopeptidases, such as cathepsin $G$ and neutrophil elastase. ${ }^{13} T$. forsythia obviously acquired this serpin gene by horizontal gene transfer from eukaryotes. ${ }^{14}$ Miropin is attached to the bacterial cell surface and also located in the periplasm exerting protection against $T$. forsythia own proteases as well as host and other bacteria enzymes, which may degrade the surface Sleyer. ${ }^{13}$ At the teeth, expression of miropin was not only strongly associated with the $T$. forsythia proteases, but also correlated with gingipains. This suggests that gingipains might be involved in regulation of miropin or vice versa. It can be speculated that the pathogenetic role of miropin is exerted by maintenance of inflammation at a low chronic level.

Correlation assessment made separately or by the principal components analysis showed differences between the teeth and implants. It is of interest to note, that significant associations between biomarkers' levels, and expression of gingipains and $T$. forsythia proteases and miropin was found only at teeth. Specifically, expression of the KLIKK proteases, as well as the protease inhibitor miropin by $T$. forsythia was correlated with gingipains. Furthermore, karilysin positively correlated with pro-inflammatory biomarkers and negatively with the anti-inflammatory cytokine IL-10. At implants there was no significant association between miropin and gingpains or between miropin and the $T$. forsythia proteases. Also no correlation was found between bacterial proteins and the analyzed biomarkers. This may suggest a substantial difference in regulation of bacterial virulence factor expression and host responses to bacteria at implants and teeth, which may partly be due to the existing anatomical differences between implants and teeth. For example, Sharpey's fibres form a complex network around the teeth together with a vascular plexus, whereas dental implants are surrounded by collagen fibres run in parallel and there are a few blood vessels ${ }^{48}$ thus the established peri-implant soft tissue resembles a scar tissue. ${ }^{49}$ Further, it has been shown that implant sites respond with a stronger inflammatory reaction than teeth sites when exposed to biofilm. ${ }^{50}$ Another factor contributing to differences may be 
related to variations in biofilm formation on the tooth and implant surface. Recently an in vitro study found that miropin expression was lower at titanium implants than at dentine disks. ${ }^{32}$ However, the miropin expression by $T$. forsythia adhered either to the dentin or titanium surface exceeded up to 500-fold the expression of proteases (miropsin-1, miropsin-2, mirolase. ${ }^{32}$ It is clear that more research is needed on $T$. forsythia, not only as the periodontal pathogen, but also a potentially pathogenic member of the peri-implant biofilm consortium.

In summary, the focus of this study was on in vivo expression (mRNA levels) of the proteases and the protease inhibitor by periodontal pathogens. Our results showed much higher expression of gingipains of $P$. gingivalis and miropin of $T$. forsythia than the KLIKK proteases of the latter bacterium. Taking into account a broad inhibitory spectrum of miropin, this data suggest that this protein may play a regulatory role in a multispecies dysbiotic biofilm forming on teeth and implant surfaces and may contribute to the initiation and/or progression of both periodontal and peri-implant diseases.

\section{Acknowledgements}

We are grateful to Christian Kindblom (previously: Department of Periodontology, Faculty of Odontology, Malmö University, Malmö, Sweden) for collecting biofilm samples and to Anna Magdoń (University of Bern, Department of Periodontology, Laboratory of Oral Microbiology) for technical assistance.

Funding: This study was funded by the participating institutions together with a grant from the European Commission (FP7-HEALTH-F3-2012-306029 "TRIGGER"). In addition, DM was supported by grant UMO-2014/15/D/NZ6/02546 from Polish National Science Center (NCN) while JP by grants from NCN (UMO-2016/21/B/NZ1/00292) and NIH NIDCR (grants R21 DE 023207 and R01 DE 022597).

Faculty of Biochemistry, Biophysics and Biotechnology of Jagiellonian University is a partner of the Leading National Research Center (KNOW) supported by the Ministry of Science and Higher Education.

All authors disclose any potential sources of conflict of interest.

\section{References}


1. Darveau RP, Hajishengallis G, Curtis MA. Porphyromonas gingivalis as a potential community activist for disease. J Dent Res. 2012;91:816-820.

2. Hajishengallis G, Darveau RP, Curtis MA. The keystone-pathogen hypothesis. Nat Rev Microbiol. 2012;10:717-725.

3. Travis J, Pike R, Imamura T, Potempa J. Porphyromonas gingivalis proteinases as virulence factors in the development of periodontitis. J Periodontal Res. 1997;32:120-125.

4. Imamura $\mathrm{T}$. The role of gingipains in the pathogenesis of periodontal disease. $J$ Periodontol. 2003;74:111-118.

5. Mikolajczyk-Pawlinska J, Kordula T, Pavloff N, et al. Genetic variation of Porphyromonas gingivalis genes encoding gingipains, cysteine proteinases with arginine or lysine specificity. Biol Chem. 1998;379:205-211.

6. Guo Y, Nguyen KA, Potempa J. Dichotomy of gingipains action as virulence factors: from cleaving substrates with the precision of a surgeon's knife to a meat chopper-like brutal degradation of proteins. Periodontol 2000. 2010;54:15-44.

7. Vincents B, Guentsch A, Kostolowska D, et al. Cleavage of IgG1 and IgG3 by gingipain K from Porphyromonas gingivalis may compromise host defense in progressive periodontitis. FASEB J. 2011;25:3741-3750.

8. Socransky SS, Haffajee AD, Cugini MA, Smith C, Kent RL, Jr. Microbial complexes in subgingival plaque. J Clin Periodontol. 1998;25:134-144.

9. Tanner AC, Kent R, Jr., Kanasi E, et al. Clinical characteristics and microbiota of progressing slight chronic periodontitis in adults. J Clin Periodontol. 2007;34:917-930.

10. Tomita S, Komiya-Ito A, Imamura K, et al. Prevalence of Aggregatibacter actinomycetemcomitans, Porphyromonas gingivalis and Tannerella forsythia in Japanese patients with generalized chronic and aggressive periodontitis. Microb Pathog. 2013;6162:11-15.

11. Ruggiero S, Cosgarea R, Potempa J, Potempa B, Eick S, Chiquet M. Cleavage of extracellular matrix in periodontitis: Gingipains differentially affect cell adhesion activities of fibronectin and tenascin-C. Biochimica Et Biophysica Acta-Molecular Basis of Disease. 2013;1832:517526.

12. Ksiazek M, Karim AY, Bryzek D, et al. Mirolase, a novel subtilisin-like serine protease from the periodontopathogen Tannerella forsythia. Biol Chem. 2015;396:261-275.

13. Ksiazek M, Mizgalska D, Enghild JJ, Scavenius C, Thogersen IB, Potempa J. Miropin, a novel bacterial serpin from the periodontopathogen Tannerella forsythia, inhibits a broad range of proteases by using different peptide bonds within the reactive center loop. J Biol Chem. 2015;290:658-670.

14. Goulas T, Ksiazek M, Garcia-Ferrer I, et al. A structure-derived snap-trap mechanism of a multispecific serpin from the dysbiotic human oral microbiome. J Biol Chem.

2017;292:10883-10898.

15. Derks J, Tomasi C. Peri-implant health and disease. A systematic review of current epidemiology. J Clin Periodontol. 2015;42 Suppl 16:S158-171.

16. Lang NP, Berglundh T, Working Group 4 of Seventh European Workshop on P. Periimplant diseases: where are we now?--Consensus of the Seventh European Workshop on Periodontology. J Clin Periodontol. 2011;38 Suppl 11:178-181.

17. Eick S, Ramseier CA, Rothenberger K, Bragger U, Buser D, Salvi GE. Microbiota at teeth and implants in partially edentulous patients. A 10-year retrospective study. Clin Oral Implants Res. 2016;27:218-225.

18. Salvi GE, Cosgarea R, Sculean A. Prevalence and Mechanisms of Peri-implant Diseases. $J$ Dent Res. 2016.

19. Armitage GC. Development of a classification system for periodontal diseases and conditions. Ann Periodontol. 1999;4:1-6.

20. Zitzmann NU, Berglundh T. Definition and prevalence of peri-implant diseases. J Clin Periodontol. 2008;35:286-291.

21. Griffiths GS. Formation, collection and significance of gingival crevice fluid. Periodontol 2000. 2003;31:32-42. 
22. Eick S, Straube A, Guentsch A, Pfister W, Jentsch H. Comparison of real-time polymerase chain reaction and DNA-strip technology in microbiological evaluation of periodontitis treatment. Diagn Microbiol Infect Dis. 2011;69:12-20.

23. Field A. Discovering statistics using SPSS. London: SAGE Publications Ltd; 2009.

24. Lee ZM, Bussema C, 3rd, Schmidt TM. rrnDB: documenting the number of rRNA and tRNA genes in bacteria and archaea. Nucleic Acids Res. 2009;37:D489-493.

25. Lynch MC, Kuramitsu HK. Role of superoxide dismutase activity in the physiology of Porphyromonas gingivalis. Infect Immun. 1999;67:3367-3375.

26. Casado PL, Canullo L, de Almeida Filardy A, Granjeiro JM, Barboza EP, Leite Duarte ME. Interleukins 1 beta and 10 expressions in the periimplant crevicular fluid from patients with untreated periimplant disease. Implant Dent. 2013;22:143-150.

27. Hultin M, Gustafsson A, Hallstrom H, Johansson LA, Ekfeldt A, Klinge B. Microbiological findings and host response in patients with peri-implantitis. Clin Oral Implants Res. 2002;13:349-358.

28. Cortelli SC, Cortelli JR, Romeiro RL, et al. Frequency of periodontal pathogens in equivalent peri-implant and periodontal clinical statuses. Arch Oral Biol. 2013;58:67-74.

29. Aoki M, Takanashi K, Matsukubo T, et al. Transmission of periodontopathic bacteria from natural teeth to implants. Clin Implant Dent Relat Res. 2012;14:406-411.

30. Salvi GE, Furst MM, Lang NP, Persson GR. One-year bacterial colonization patterns of Staphylococcus aureus and other bacteria at implants and adjacent teeth. Clin Oral Implants Res. 2008;19:242-248.

31. Quirynen M, Papaioannou W, van Steenberghe D. Intraoral transmission and the colonization of oral hard surfaces. J Periodontol. 1996;67:986-993.

32. Eick S, Kindblom C, Mizgalska D, et al. Adhesion of Porphyromonas gingivalis and Tannerella forsythia to dentin and titanium with sandblasted and acid etched surface coated with serum and serum proteins - An in vitro study. Arch Oral Biol. 2017;75:81-88.

33. Guentsch A, Hirsch C, Pfister W, et al. Cleavage of IgG1 in gingival crevicular fluid is associated with the presence of Porphyromonas gingivalis. J Periodontal Res. 2013;48:458465.

34. Guentsch A, Kramesberger M, Sroka A, Pfister W, Potempa J, Eick S. Comparison of gingival crevicular fluid sampling methods in patients with severe chronic periodontitis. $J$ Periodontol. 2011;82:1051-1060.

35. Guo M, Wang Z, Fan X, et al. kgp, rgpA, and rgpB DNA vaccines induce antibody responses in experimental peri-implantitis. J Periodontol. 2014;85:1575-1581.

36. Katz J, Yang QB, Zhang P, et al. Hydrolysis of epithelial junctional proteins by Porphyromonas gingivalis gingipains. Infect Immun. 2002;70:2512-2518.

37. Sochalska M, Potempa J. Manipulation of Neutrophils by Porphyromonas gingivalis in the Development of Periodontitis. Front Cell Infect Microbiol. 2017;7:197.

38. Jusko M, Potempa J, Karim AY, et al. A metalloproteinase karilysin present in the majority of Tannerella forsythia isolates inhibits all pathways of the complement system. J Immunol. 2012;188:2338-2349.

39. Jusko M, Potempa J, Mizgalska D, et al. A metalloproteinase mirolysin of Tannerella forsythia inhibits all pathways of the complement system. J Immunol. 2015;195:2231-2240.

40. Ksiazek M, Mizgalska D, Eick S, Thogersen IB, Enghild JJ, Potempa J. KLIKK proteases of Tannerella forsythia: putative virulence factors with a unique domain structure. Front Microbiol. 2015;6:312.

41. Karim AY, Kulczycka M, Kantyka T, et al. A novel matrix metalloprotease-like enzyme (karilysin) of the periodontal pathogen Tannerella forsythia ATCC 43037. Biol Chem. 2010;391:105-117.

42. Koziel J, Karim AY, Przybyszewska K, et al. Proteolytic inactivation of LL-37 by karilysin, a novel virulence mechanism of Tannerella forsythia. J Innate Immun. 2010;2:288-293.

43. Bryzek D, Ksiazek M, Bielecka E, et al. A pathogenic trace of Tannerella forsythia - shedding of soluble fully active tumor necrosis factor alpha from the macrophage surface by karilysin. Mol Oral Microbiol. 2014;29:294-306. 
44. Koneru L, Ksiazek M, Waligorska I, et al. Mirolysin, a LysargiNase from Tannerella forsythia, proteolytically inactivates the human cathelicidin, LL-37. Biol Chem. 2016.

45. Tamai R, Deng X, Kiyoura Y. Porphyromonas gingivalis with either Tannerella forsythia or Treponema denticola induces synergistic IL-6 production by murine macrophage-like J774.1 cells. Anaerobe. 2009;15:87-90.

46. Jung YJ, Jun HK, Choi BK. Gingipain-dependent augmentation by Porphyromonas gingivalis of phagocytosis of Tannerella forsythia. Mol Oral Microbiol. 2016;31:457-471.

47. Mkaouar H, Akermi N, Mariaule V, et al. Siropins, novel serine protease inhibitors from gut microbiota acting on human proteases involved in inflammatory bowel diseases. Microb Cell Fact. 2016;15:201.

48. Thoma DS, Muhlemann S, Jung RE. Critical soft-tissue dimensions with dental implants and treatment concepts. Periodontol 2000. 2014;66:106-118.

49. Sculean A, Gruber R, Bosshardt DD. Soft tissue wound healing around teeth and dental implants. J Clin Periodontol. 2014;41 Suppl 15:S6-22.

50. Salvi GE, Aglietta M, Eick S, Sculean A, Lang NP, Ramseier CA. Reversibility of experimental peri-implant mucositis compared with experimental gingivitis in humans. Clin Oral Implants Res. 2012;23:182-190.

51. Frohlich E, Kantyka T, Plaza K, et al. Benzamidine derivatives inhibit the virulence of Porphyromonas gingivalis. Molecular Oral Microbiology. 2013;28:192-203. 
Table 1

Primer pairs used in real-time PCR for detection of bacterial proteases (rgpA, rgpB, kgp, miropsin-1, miropsin-2, mirolase) and of T. forsythia protease inhibitor (miropin) as well as the house keeping genes (sod)

\begin{tabular}{|c|c|c|c|}
\hline Species & Gene & Primer & Reference \\
\hline \multirow[t]{4}{*}{ P. gingivalis } & $\operatorname{rgp} A$ & $\begin{array}{l}\text { fwd: 5'-TAT CCT TCG TGA TGT GCG TG-3' } \\
\text { rev: 5'-GCT GTA ACG GGA GAA GCA AT-3' }\end{array}$ & Frohlich et al. ${ }^{51}$ \\
\hline & $\operatorname{rgp} B$ & $\begin{array}{l}\text { fwd: 5'-CAT TCT CCT CTC TGT TGG GA-3' rev: 5'- } \\
\text { CGT AGG GGA TTT GAT CAG GA-3' }\end{array}$ & Frohlich et al. ${ }^{51}$ \\
\hline & $k g p$ & $\begin{array}{l}\text { fwd: 5'-TCA AGC AGT TCG ATG CAA GC -3' } \\
\text { rev: 5'-ACT TGG GTC AGT TCT TGT CC-3' }\end{array}$ & Frohlich et al. ${ }^{51}$ \\
\hline & sod & $\begin{array}{l}\text { fwd: 5'-AAT TCC ACC ACG GTA AGC AC-3' } \\
\text { 5'- TTC TCG ATG GAC AGT TTG CC-3' }\end{array}$ & Frohlich et al..$^{51}$ \\
\hline \multirow[t]{5}{*}{ T. forsythia } & miropsin-1 & $\begin{array}{l}\text { fwd: 5'-CGT GCG TGA AGA AGC CAT TA-3' } \\
\text { rev: 5'-AAC CCG GAT GTT CAT ACC CC-3' }\end{array}$ & according to Ksiazek et al. ${ }^{40}$ \\
\hline & miropsin-2 & $\begin{array}{l}\text { fwd: 5'-TCC TGA CCG ACC TGA TCA AA-3' } \\
\text { rev: 5'-TCG GCA TTG GAA ATT TCG GA-3' }\end{array}$ & according to Ksiazek et al. ${ }^{40}$ \\
\hline & karilysin & $\begin{array}{l}\text { fwd: 5'- TTA CAG TTG CGG CAC ATG AG-3' } \\
\text { rev: 5'- TGT TAA TGG TTG CTC GCA CT -3' }\end{array}$ & accession: Tf 0367; BFO2683 \\
\hline & mirolysin & $\begin{array}{l}\text { fwd: 5'- CGA ACA TCG ACT TCC ACA GA-3' } \\
\text { rev: 5'- TGT TTT AGG GAA CGA AGG ACA -3' }\end{array}$ & accession: Tf0341; BFO2661 \\
\hline & mirolase & $\begin{array}{l}\text { fwd: 5'-TGC CGC AAA TCA TAA TGG TA } \\
\text { rev: 5'-GTC CAT CCC TTC CTT GAG TG-3' }\end{array}$ & according to Ksiazek et al. ${ }^{40}$ \\
\hline
\end{tabular}



rev: 5'- TCT AGT AAT TTG TTC TCC AAT TTG C -3'

miropin fwd: 5'-ATG CCT TTG CCT TCG ATC TG-3' according to Ksiazek et al. ${ }^{13}$ rev: 5“-CTT CCC GTA GTG AAT GGC TG-3’ 
Table 2

Presence of selected species in subgingival and peri-implant biofilm

\begin{tabular}{|c|c|c|c|c|c|c|}
\hline \multirow[t]{2}{*}{ Species } & \multicolumn{2}{|l|}{ Healthy } & \multicolumn{2}{|c|}{ gingivitis / mucositis } & \multicolumn{2}{|c|}{ periodontitis / peri-implantitis } \\
\hline & tooth $(n=10)$ & implant $(n=10)$ & tooth $(n=12)$ & implant $(n=12)$ & tooth $(n=10)$ & implant $(n=10)$ \\
\hline A. actinomycetemcomitans & $1(10.0)$ & $2(20.0)$ & $3(25.0)$ & $3(25.0)$ & $3(30.0)$ & $3(30.0)$ \\
\hline T. forsythia & $2(20.0)$ & $4(40.0)$ & $5(41.7)$ & $6(50.0)$ & $8(80.0)$ & $10(100)$ \\
\hline T. denticola & $1(10.0)$ & $3(30.0)$ & $5(41.7)$ & $6(50.0)$ & $8(80.0)$ & $10(100)$ \\
\hline
\end{tabular}


Table 3

DNA (dichotomized results) and mRNA expression of gingipains in samples with less (dichotomized results) and equal or more than $10^{5}$ (dichotomized results and quantitative data related to sod) Porphyromonas gingivalis

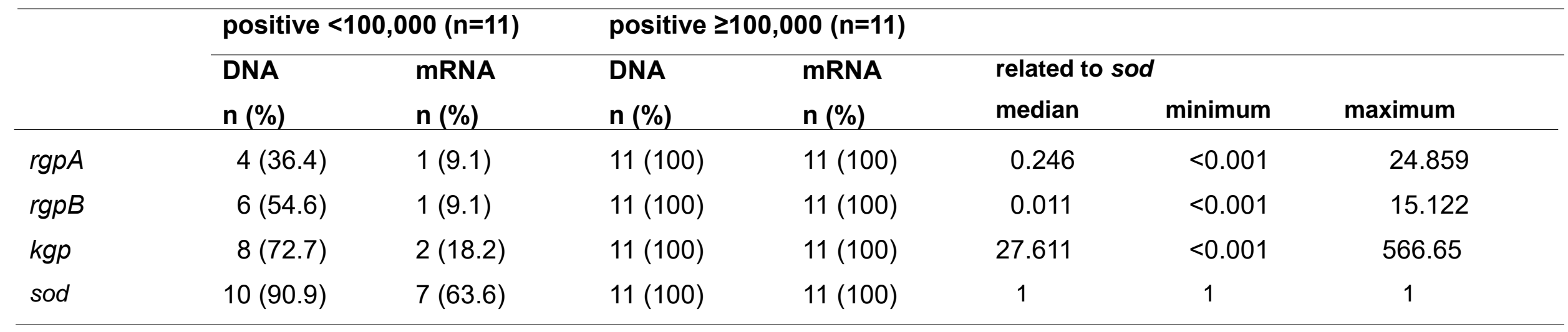


Table 4

DNA (dichotomized results) and mRNA expression of Tannerella forsythia proteases and miropin in samples with less (dichotomized results) and equal or more than (dichotomized results and quantitative data related to sod) $10^{5} \mathrm{~T}$. forsythia

\begin{tabular}{|c|c|c|c|c|c|c|c|}
\hline & \multicolumn{2}{|c|}{ positive $<100,000(n=6)$} & \multicolumn{2}{|c|}{ positive $\geq 100,000(n=29)$} & & \multirow[b]{2}{*}{ minimum } & \multirow[b]{2}{*}{ maximum } \\
\hline & $\begin{array}{l}\text { DNA } \\
\text { n (\%) }\end{array}$ & $\begin{array}{l}\text { mRNA } \\
\text { n (\%) }\end{array}$ & $\begin{array}{l}\text { DNA } \\
\text { n (\%) }\end{array}$ & $\begin{array}{l}\text { mRNA } \\
\mathrm{n}(\%)\end{array}$ & $\begin{array}{l}\text { related to sod } \\
\text { median }\end{array}$ & & \\
\hline miropsin-2 & $3(50.0)$ & $0(0)$ & $29(100)$ & $14(48.3)$ & 0.001 & 0.000 & 3.051 \\
\hline karilysin & $4(66.7)$ & $1(16.7)$ & $21(72.4)$ & $11(37.9)$ & 0.000 & 0.000 & 0.792 \\
\hline mirolase & $4(66.7)$ & $0(0)$ & $29(100)$ & $15(51.7)$ & 0.006 & 0.000 & 0.380 \\
\hline forsylisin-1 & $0(0)$ & $0(0)$ & $25(86.2)$ & $6(20.7)$ & 0.000 & 0.000 & 0.012 \\
\hline miropin & $6(100)$ & $6(100)$ & $29(100)$ & $29(100)$ & 2.340 & $<0.001$ & 17.609 \\
\hline sod & $6(100)$ & $3(50)$ & $29(100)$ & $29(100)$ & 1 & 1 & 1 \\
\hline
\end{tabular}


Table 5

mRNA expression (dichotomized results) of Tannerella forsythia proteases and miropin related to clinical data

\begin{tabular}{|c|c|c|c|c|c|c|}
\hline & \multicolumn{3}{|l|}{ Tooth } & \multicolumn{3}{|l|}{ implant } \\
\hline & $\begin{array}{l}\text { healthy } \\
\mathrm{n}(\%)\end{array}$ & $\begin{array}{l}\text { gingivitis } \\
\mathrm{n}(\%)\end{array}$ & $\begin{array}{l}\text { periodontitis } \\
\mathrm{n}(\%)\end{array}$ & $\begin{array}{l}\text { healthy } \\
\mathrm{n}(\%)\end{array}$ & $\begin{array}{l}\text { mucositis } \\
\mathrm{n}(\%)\end{array}$ & $\begin{array}{l}\text { peri-implantitis } \\
\mathrm{n}(\%)\end{array}$ \\
\hline miropsin-1 & $1(50.0)$ & $2(50.0)$ & 7 (87.5) & $0(0)$ & $2(50.0)$ & $5(55.6)$ \\
\hline miropsin-2 & $0(0)$ & $2(50.0)$ & 7 (87.5) & $0(0)$ & $0(0)$ & $5(55.6)$ \\
\hline mirolysin & $1(50.0)$ & $1(25.0)$ & $3(37.5)$ & $0(0)$ & $1(25.0)$ & $5(55.6)$ \\
\hline mirolase & $0(0)$ & $2(50.0)$ & $6(75.0)$ & $0(0)$ & $1(25.0)$ & $6(66.7)$ \\
\hline forsylisin-1 & $0(0)$ & $0(0)$ & $3(37.5)$ & $0(0)$ & $1(25.0)$ & 2 (22.2) \\
\hline miropin & $2(100)$ & $4(100)$ & $8(100)$ & $2(100)$ & $4(100)$ & $9(100)$ \\
\hline
\end{tabular}

(only samples with $T$. forsythia $\geq 10^{5}$ ) 
Table 6

Principal components analysis (loadings of the main components) at teeth and implants

\begin{tabular}{|c|c|c|c|c|}
\hline \multirow[t]{2}{*}{ Variable } & \multicolumn{2}{|c|}{ Principal component teeth } & \multicolumn{2}{|c|}{ Principal component implants } \\
\hline & Component 1 & Component 2 & Component 1 & Component 2 \\
\hline $\operatorname{rgp} A$ & 0.616 & 0 & 0 & 0.939 \\
\hline $\operatorname{rgp} B$ & 0 & 0 & 0 & 0.937 \\
\hline kgp & 0 & 0 & 0 & 0.885 \\
\hline miropsin-1 & 0.743 & 0 & 0 & 0 \\
\hline miropsin-2 & 0 & 0 & 0.873 & 0 \\
\hline karilysin & 0 & 0.645 & 0 & 0 \\
\hline mirolysin & 0 & 0 & 0.708 & 0 \\
\hline mirolase & 0.906 & 0 & 0.910 & 0 \\
\hline forsylisin-1 & 0.877 & 0 & 0.855 & 0 \\
\hline miropin & 0.741 & 0 & 0 & 0 \\
\hline IL-8 & 0 & 0.549 & 0 & 0 \\
\hline IL-1 $\beta$ & 0 & 0.867 & 0 & 0 \\
\hline IL-10 & 0 & -0.600 & 0 & 0 \\
\hline$\%$ of variance & 25.23 & 21.00 & 25.57 & 23.09 \\
\hline
\end{tabular}

Components with a variance $\geq 20 \%$ are presented. Loadings of the principal components $<|0.5|$ are set to 0 for greater clarity. 
(i)

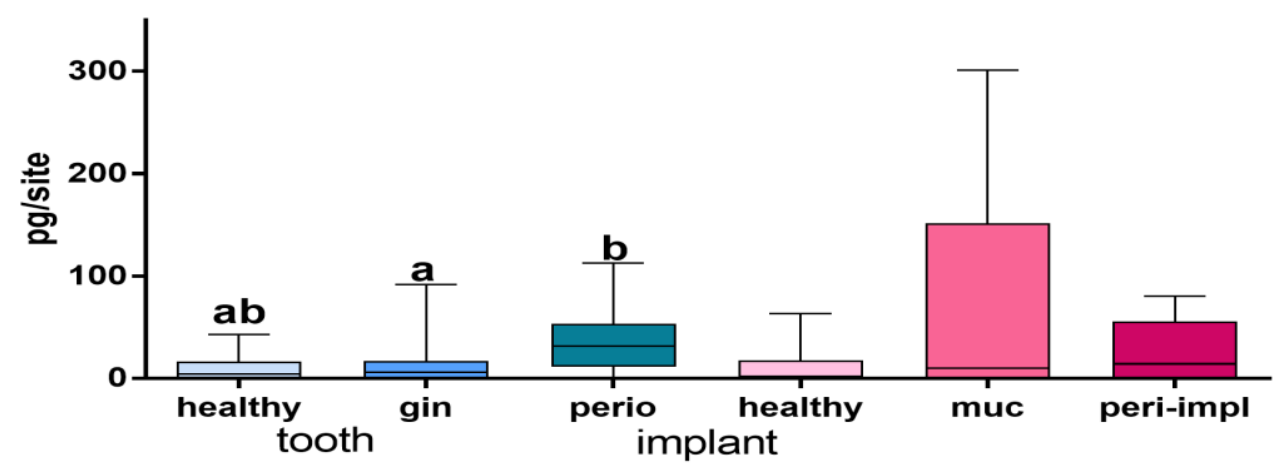

(ii)

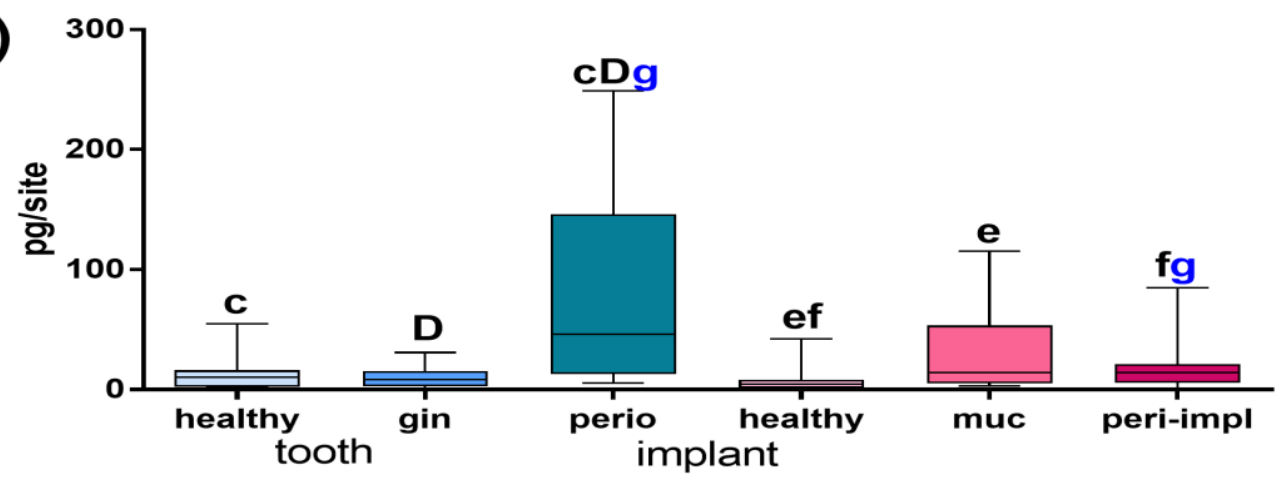

(iii)

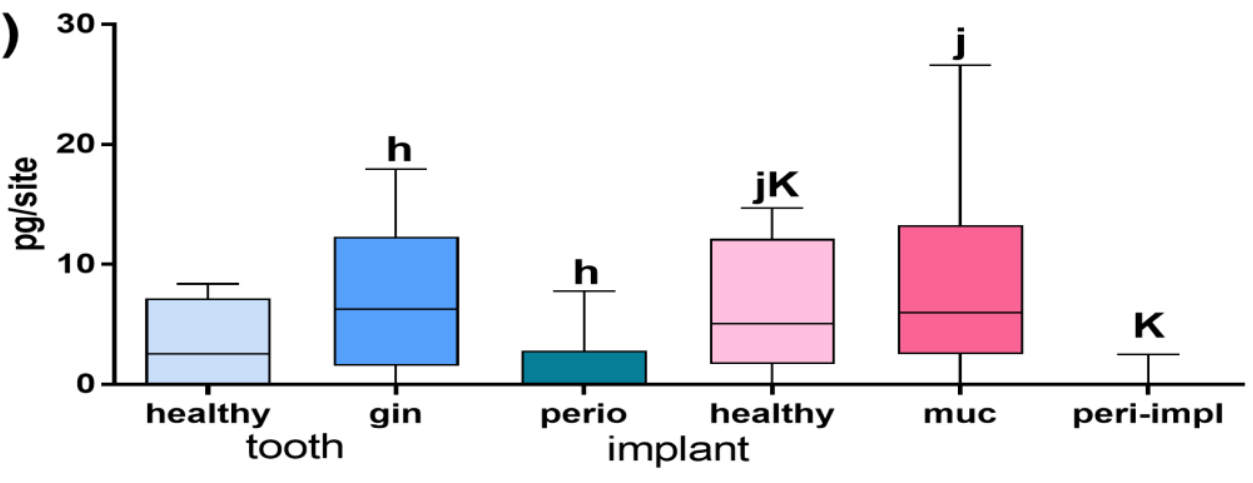

Comparison between two groups

at teeth: a, b, c, h $(p<0.05) ; \mathbf{D}(p<0.01)$

at implants: e, f, j $(p<0.05) ; \mathbf{K}(p<0.01)$

at teeth and implants: $g(p<0.05)$

\section{Figure 1}

Levels of the chemokine interleukin (IL)-8 (i), and of the cytokines IL-1 $\beta$ (ii) and IL-10 (iii) at teeth being periodontally healthy (healthy), with gingivitis (gin) and with periodontitis (perio) sites as well as at implants being healthy, with mucositis (muc) and with peri-implantitis (periimpl)

Statistical analysis compared cytokines levels at teeth and at implants as well as between the healthy sites and sites with inflammation (gingivitis, mucositis) and between sites with alveolar bone loss (periodontitis, peri-implantitis). 